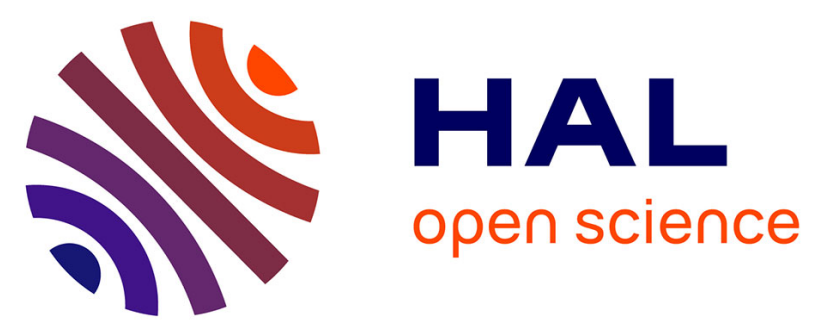

\title{
Influence of a Ta spacer on the magnetic and transport properties of perpendicular magnetic tunnel junctions
}

Léa Cuchet, Bernard Rodmacq, Stéphane Auffret, Ricardo C. Sousa, Clarisse Ducruet, Bernard Dieny

\section{- To cite this version:}

Léa Cuchet, Bernard Rodmacq, Stéphane Auffret, Ricardo C. Sousa, Clarisse Ducruet, et al.. Influence of a Ta spacer on the magnetic and transport properties of perpendicular magnetic tunnel junctions. Applied Physics Letters, 2013, 103 (5), pp.052402. 10.1063/1.4816968 . cea-01073021

\section{HAL Id: cea-01073021} https://hal-cea.archives-ouvertes.fr/cea-01073021

Submitted on 31 Aug 2021

HAL is a multi-disciplinary open access archive for the deposit and dissemination of scientific research documents, whether they are published or not. The documents may come from teaching and research institutions in France or abroad, or from public or private research centers.
L'archive ouverte pluridisciplinaire HAL, est destinée au dépôt et à la diffusion de documents scientifiques de niveau recherche, publiés ou non, émanant des établissements d'enseignement et de recherche français ou étrangers, des laboratoires publics ou privés. 


\title{
Influence of a Ta spacer on the magnetic and transport properties of perpendicular magnetic tunnel junctions
}

\author{
Léa Cuchet, Bernard Rodmacq, Stéphane Auffret, Ricardo C. Sousa, Clarisse Ducruet, \\ and Bernard Dieny \\ SPINTEC, UMR8191, CEA/CNRS/UJF/G-INP, INAC-CEA Grenoble, 38054 Grenoble Cedex, France
}

(Received 25 June 2013; accepted 14 July 2013; published online 29 July 2013)

\begin{abstract}
Ultrathin Ta layers were inserted in the bottom hard $(\mathrm{Co} / \mathrm{Pt}) / \mathrm{Ta} / \mathrm{CoFeB} / \mathrm{MgO}$ magnetic electrode of perpendicular magnetic tunnel junctions. The magnetization of the top part of this electrode abruptly falls in-plane when the Ta thickness exceeds $0.45 \mathrm{~nm}$. This results from the balance between the various energy terms acting on this layer (exchange-like coupling through Ta, demagnetizing energy, and perpendicular anisotropy at the $\mathrm{CoFeB} / \mathrm{MgO}$ interface). For small $\mathrm{Ta}$ thicknesses, this insertion leads to a strong improvement of the tunnel magnetoresistance, as long as the magnetization of all layers remains perpendicular-to-plane. (C) 2013 AIP Publishing LLC. [http://dx.doi.org/10.1063/1.4816968]
\end{abstract}

Spin Transfer Torque Random Access Memories (STTRAM) represent a promising type of non-volatile memories that are particularly attractive because of their unique combination of qualities: high write speed (a few ns), low write energy (a few pJ), infinite endurance $\left(>10^{16}\right.$ write cycles), small size $\left(\sim 6 \mathrm{~F}^{2}, \mathrm{~F}\right.$ being the feature size associated with the technology node). A lot of work has been carried out on Magnetic Tunnel Junctions (MTJs), since first-principle calculations predicted in $2001^{1}$ that a very large Tunnel MagnetoResistance (TMR) ratio could be reached for junctions based on a crystalline $\mathrm{MgO}$ insulating barrier and bcc $\mathrm{Fe}$ or $\mathrm{FeCo}$ magnetic electrodes. This phenomenon was experimentally evidenced in 2004 for $\mathrm{Fe} / \mathrm{MgO} / \mathrm{Fe}$ epitaxial structures $^{2}$ as well as for $\mathrm{CoFe} / \mathrm{MgO} / \mathrm{CoFe}$ sputtered junctions, ${ }^{3}$ with a TMR ratio around $180 \%$. Later on, due to an easier implementation, research focused on sputtered MTJs comprising amorphous $\mathrm{CoFeB}$ electrodes that have the advantage, after annealing, to yield the right bcc (100) texture. ${ }^{4}$ These structures enabled getting a TMR ratio of the order of a few hundreds of percent.

Initially, these junctions were based on magnetic electrodes with in-plane magnetization, for which the stability of the magnetic coding is achieved, through shape anisotropy, by giving to the memory cell an elliptical shape (typical aspect ratio of the order of 2 to 2.5). However, the resulting energy barrier only yields sufficient thermal stability down to cell size of the order of $65 \times 170 \mathrm{~nm}^{2}$. More recently, MTJs with perpendicularly magnetized electrodes (p-MTJs) have attracted a lot of interest. This is due to the expected higher storage densities and thermal stability which can be achieved with this kind of structures. Besides, for a given retention time of the information, it was shown that lower current densities are needed with p-MTJs written by STT switching compared to in-plane magnetized MTJs. ${ }^{5}$ To introduce perpendicular magnetic anisotropy (PMA) in such tunnel junctions, one may use $\mathrm{Co} / \mathrm{Pt}$ or $\mathrm{Co} / \mathrm{Pd}$ multilayers. ${ }^{6,7} \mathrm{In}$ addition, it was shown more than ten years ago that the magnetic metal/oxide interface also contributes to PMA, ${ }^{8,9}$ and more recently that the interfacial properties of both seed and capping layers can have a significant impact on PMA. ${ }^{6,10}$
Even if $\mathrm{Co} / \mathrm{Pt}(\mathrm{Pd})$ multilayers are a good choice to induce or reinforce PMA in CoFeB layers by exchange-like coupling, it is necessary to introduce a spacer between both magnetic layers. ${ }^{6,11}$ Indeed, it is very difficult to get the right bcc (100) texture for $\mathrm{CoFeB}$ electrodes when they directly grow on fcc (111) Pt (Pd) or hcp (0001) Co layers. The role of this spacer is thus to structurally decouple the two magnetic entities ( $\mathrm{Co} / \mathrm{Pt}$ multilayer and $\mathrm{CoFeB}$ layer), thus allowing crystallization of the $\mathrm{CoFeB}$ layers from the $\mathrm{MgO}$ side with the adequate bcc (100) texture. In addition, this spacer has a second beneficial effect, since it attracts, upon annealing, boron atoms out of the $\mathrm{CoFeB}$ layer and away from the $\mathrm{CoFeB} / \mathrm{MgO}$ interface. ${ }^{12}$ However, if this spacer gets too thick, it also magnetically decouples the magnetic layers, so that the PMA coming from the $(\mathrm{Pt} / \mathrm{Co})$ multilayer can no longer help pulling the $\mathrm{CoFeB}$ magnetization outof-plane. Sokalski et al. ${ }^{13}$ recently showed on $\mathrm{FeCoB} / \mathrm{Ta} /$ $\mathrm{FeCoB} / \mathrm{MgO}$ electrodes that it is possible to find a window of Ta thickness allowing both strong PMA and strong ferromagnetic coupling between the two FeCoB layers. However, in that case PMA is much smaller than that obtained thanks to exchange coupling to $\mathrm{Co} / \mathrm{Pt}(\mathrm{Pd})$ multilayers.

In this letter, we present the effect of a thin Ta insertion layer in the bottom hard electrode of perpendicular tunnel junctions on their magnetic and transport properties. In that hard electrode, the perpendicular $\mathrm{Co} / \mathrm{Pt}$ multilayer is exchange-coupled to a $\mathrm{CoFeB}$ layer through this thin $\mathrm{Ta}$ layer of varying thickness. We show that the Ta insertion initially greatly improves the TMR ratio, up to a critical thickness of $0.45 \mathrm{~nm}$, above which magnetic decoupling through Ta leads to a reorientation of the $\mathrm{CoFeB}$ magnetization from out-of-plane to in-plane, with a corresponding abrupt decrease of the TMR ratio.

Samples were deposited by magnetron sputtering under an Ar pressure of $2 \cdot 10^{-3}$ millibar. The stack of the junctions is the following (thicknesses are given in $\mathrm{nm}$ ): $\mathrm{Si} / \mathrm{SiO}_{2} / \mathrm{Ta} 3 /$ $\mathrm{Pt} 5 /(\mathrm{Co} 0.5 / \mathrm{Pt} 0.4)_{5} / \mathrm{Co} 0.5 / \mathrm{Tax}_{\mathrm{Ta}} / \mathrm{Co}_{60} \mathrm{Fe}_{20} \mathrm{~B}_{20} 1.2 / \mathrm{MgO} / \mathrm{Fe}_{72} \mathrm{Co}_{8}$ $\mathrm{B}_{20} 1.2 / \mathrm{Ta} 1 / \mathrm{Pt} 2$, where the Ta thickness $\mathrm{x}_{\mathrm{Ta}}$ varies between 0 and $0.9 \mathrm{~nm}$. We use two compositions of amorphous $\mathrm{CoFeB}$ alloy that we will later call $\mathrm{CoFeB}$ and $\mathrm{FeCoB}$, for Co-rich 
and Fe-rich alloys, respectively. The $\mathrm{MgO}$ barrier is obtained by natural oxidation of a $0.9 \mathrm{~nm}$ thick metallic layer of $\mathrm{Mg}$ under an oxygen pressure of $150 \mathrm{mbar}$, for $10 \mathrm{~s}$. On top of this oxidized layer, a second layer of $0.5 \mathrm{~nm}$ of $\mathrm{Mg}$ is then deposited. Samples were annealed under $10^{-6}$ millibar vacuum at $300^{\circ} \mathrm{C}$ for $1 \mathrm{~h}$ without applied magnetic field. Magnetic characterizations were performed using an Extraordinary Hall Effect (EHE) setup and by Vibrating Sample Magnetometry (VSM), the magnetic field being applied perpendicular to the sample plane. We remind that the extraordinary part of the Hall resistance is proportional to the perpendicular component of the magnetization. Macroscopic transport measurements were performed using the CIPT technique ${ }^{14}$ on identical samples deposited in the same run on a $60 \mathrm{~nm}$ thick $\mathrm{CuN}$ buffer layer and subsequently covered by a $15 \mathrm{~nm}$ thick Ru layer, in order to adapt the resistance of bottom and top electrodes to that of the $\mathrm{MgO}$ barrier.

A typical magnetic hysteresis loop without Ta insertion $\left(\mathrm{x}_{\mathrm{Ta}}=0\right)$ is shown in Figure 1. Transitions of the top magnetically soft $\mathrm{FeCoB}$ and the bottom hard electrodes are visible, both of them exhibiting perpendicular anisotropy. A minor loop is realized on the top electrode and the corresponding curve is shown in red in Figure 1. It can be seen that this loop is not centered on zero field but is slightly shifted towards positive fields (coming from positive saturation), showing that an antiferromagnetic coupling exists through the $\mathrm{MgO}$ barrier between the two magnetic electrodes. Such an antiferromagnetic coupling has already been observed in perpendicular magnetic junctions, and arises from Néel-type coupling for structures with strong perpendicular anisotropy. ${ }^{15,16}$ For this sample without Ta insertion, the coupling field $\mathrm{H}_{\mathrm{cpl}}$ amounts to $-80 \mathrm{Oe}$, with the usual sign convention.

The same measurements were performed for increasing values of the Ta insertion thickness $\mathrm{x}_{\mathrm{Ta}}$ and Figure 2 shows the corresponding loops for $\mathrm{x}_{\mathrm{Ta}}=0.4,0.5$, and $0.6 \mathrm{~nm}$. In order to facilitate the comparison between samples, the three curves are normalized to their maximum Hall resistance value, and only part of the hysteresis cycle (from $+4 \mathrm{kOe}$ to $-1 \mathrm{kOe}$ ) is shown. One observes the appearance of a hard axis component in the loops for $\mathrm{x}_{\mathrm{Ta}}=0.5$ and $0.6 \mathrm{~nm}$. This component is attributed to the $\mathrm{CoFeB}$ layer deposited on top

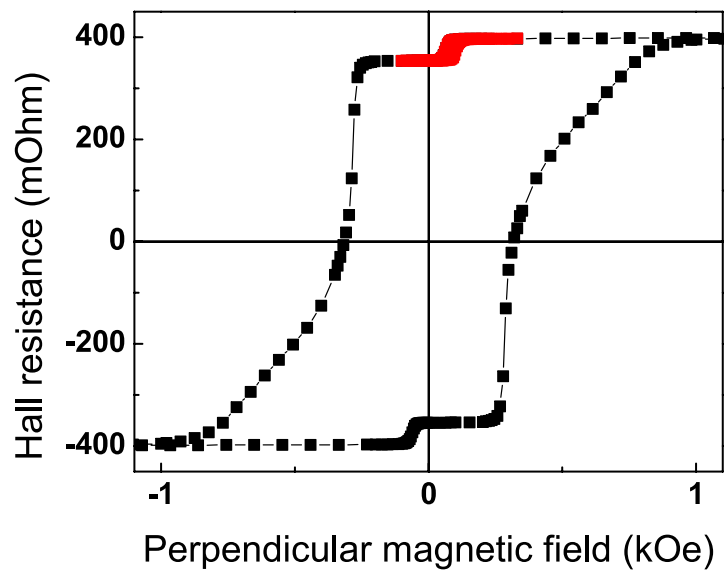

FIG. 1. Typical EHE loop for $\mathrm{x}_{\mathrm{Ta}}=0 \mathrm{~nm}$. The red curve shows the minor cycle of the soft layer.

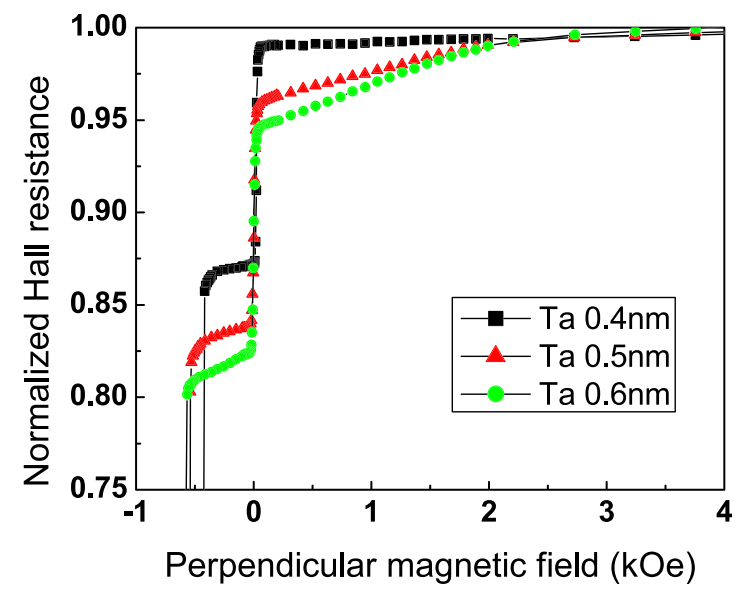

FIG. 2. Part of the EHE hysteresis loops (between +4 and $-1 \mathrm{kOe}$ ) for $\mathrm{x}_{\mathrm{Ta}}=0.4,0.5$, and $0.6 \mathrm{~nm}$.

of the Ta spacer, whose magnetization abruptly decouples from that of the bottom $\mathrm{Co} / \mathrm{Pt}$ multilayer and falls in-plane. The field required to pull back this layer in the perpendicular direction amounts to about $3 \mathrm{kOe}$. This reorientation of the anisotropy direction is not observed in the work of Sokalski et al. ${ }^{13}$ since in their case the CoFeB layer on top of the Ta insertion is thin enough to keep perpendicular anisotropy by itself, even when it is fully decoupled from the $\mathrm{CoFeB}$ layer deposited below the Ta layer. However, the critical Ta thickness they quote is similar to the one we observe in our samples.

Figure 3 presents the variation of the amplitude of this in-plane signal (normalized to the Hall amplitude of the bottom electrode) as a function of the Ta insertion thickness. For thicknesses up to $0.45 \mathrm{~nm}$, no in-plane component is visible on the hysteresis loop, whereas for thicknesses above $0.55 \mathrm{~nm}$ this component becomes constant with an amplitude of about $4.5 \%$ of the Hall signal. For $\mathrm{x}_{\mathrm{Ta}}=0.5 \mathrm{~nm}$, the constant level of amplitude is not yet reached which leads us to assume that the system is in an intermediate state for this thickness: magnetization is not homogeneous in the whole $\mathrm{CoFeB}$ layer and some parts (probably because of the

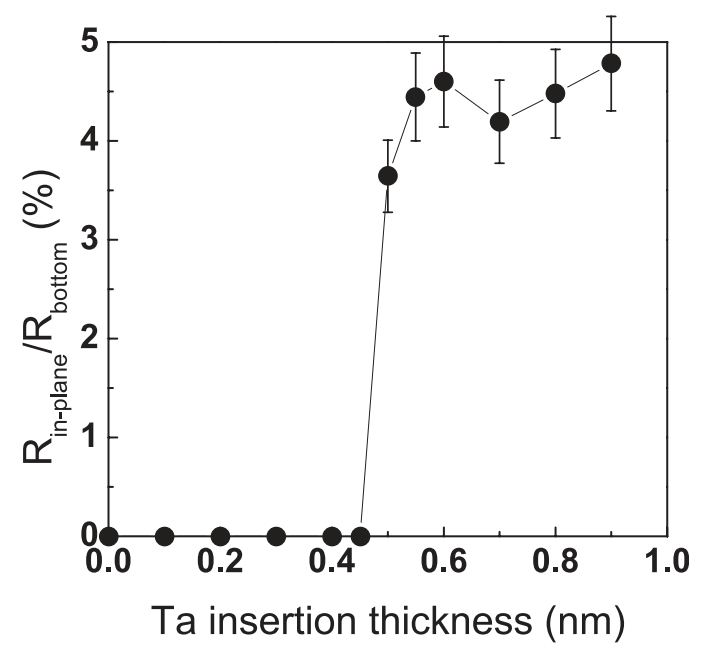

FIG. 3. Relative Hall contribution of the in-plane component of the bottom electrode as a function of the Ta insertion thickness. 
roughness of the layers) remain with a perpendicular anisotropy. Let us note that the Hall amplitude of the in-plane component is much smaller than its expected magnetization contribution (about 30\% assuming equal $\mathrm{Co}$ and $\mathrm{CoFeB}$ magnetizations). This comes from the fact that the Hall coefficient of the $\mathrm{Ta} / \mathrm{CoFeB} / \mathrm{MgO}$ layer is much smaller than that of the $\mathrm{Pt} / \mathrm{Co} / \mathrm{Pt}$ ones due to a weaker spin-orbit coupling in the $\mathrm{CoFeB}$ layer as compared to the $(\mathrm{Co} / \mathrm{Pt})$ multilayer. However, complementary VSM measurements give the expected relative magnetic contribution of both perpendicular and in-plane components of the bottom magnetic electrode on both sides of the Ta insertion layer.

Figure 4(a) shows the variation of the anisotropy field of the in-plane contribution as a function of the Ta insertion thickness (i.e., the field needed to orient perpendicularly the magnetization of the in-plane $\mathrm{CoFeB}$ layer). Following the usual convention, this field is given with a negative sign. As we have shown before, this in-plane contribution appears for $\mathrm{x}_{\mathrm{Ta}}=0.5 \mathrm{~nm}$. The anisotropy field then progressively decreases with increasing Ta thickness, from about $-3 \mathrm{kOe}$ for $\mathrm{x}_{\mathrm{Ta}}=0.5 \mathrm{~nm}$ to $-1 \mathrm{kOe}$ for $\mathrm{x}_{\mathrm{Ta}}=0.9 \mathrm{~nm}$. This can be explained by an increasing perpendicular anisotropy of the in-plane magnetized $\mathrm{CoFeB}$ layer deposited on a Ta layer of increasing thickness. Ta presents the ability to attract boron from $\mathrm{CoFeB}$ during annealing. ${ }^{12}$ As the boron atoms diffuse away from the $\mathrm{CoFeB} / \mathrm{MgO}$ interface, PMA is further improved. ${ }^{10,17,18}$ Thus, when the thickness of the Ta insertion increases, it becomes easier to reorient the in-plane magnetized $\mathrm{CoFeB}$ layer towards the perpendicular direction. Although papers report some PMA contribution of the Ta/ $\mathrm{CoFeB}$ interface, ${ }^{6,10}$ others indicate that PMA solely comes from the $\mathrm{CoFeB} / \mathrm{MgO}$ one, this PMA being enhanced by a better growth on that Ta seed layer. ${ }^{19}$ The constant relative Hall amplitude above $\mathrm{x}_{\mathrm{Ta}}=0.55 \mathrm{~nm}$ observed in Figure 3, confirmed by VSM measurements, excludes the possibility of a progressive alloying between the Ta and $\mathrm{CoFeB}$ layers, which would lead to a decrease of the effective magnetic thickness and thus to a decrease of the anisotropy field.

As already pointed out in Figure 1, there is antiferromagnetic coupling between the magnetic electrodes through the $\mathrm{MgO}$ barrier. The evolution of this parameter is representative of the effect of both increasing Ta layer thickness and magnetic anisotropy reorientation of the bottom $\mathrm{CoFeB}$

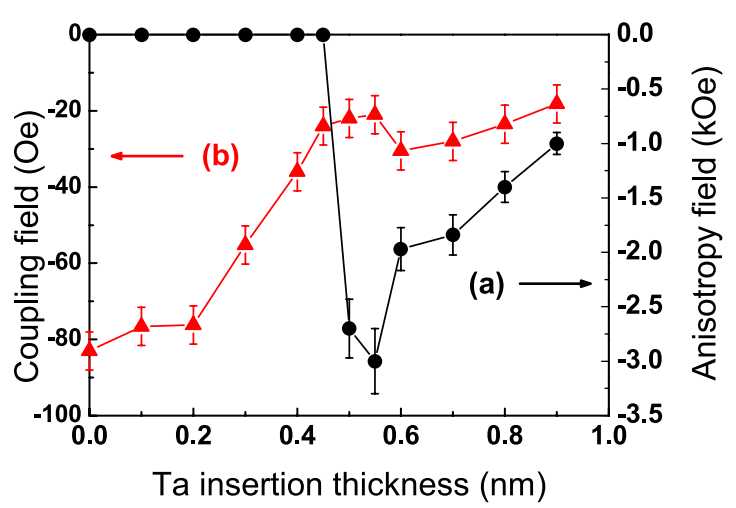

FIG. 4. Variation as a function of the Ta insertion thickness of (a) the perpendicular anisotropy field of the in-plane contribution (black circles, righthand scale) and (b) the coupling field between the bottom and top magnetic layers through the $\mathrm{MgO}$ barrier (red triangles, left-hand scale).

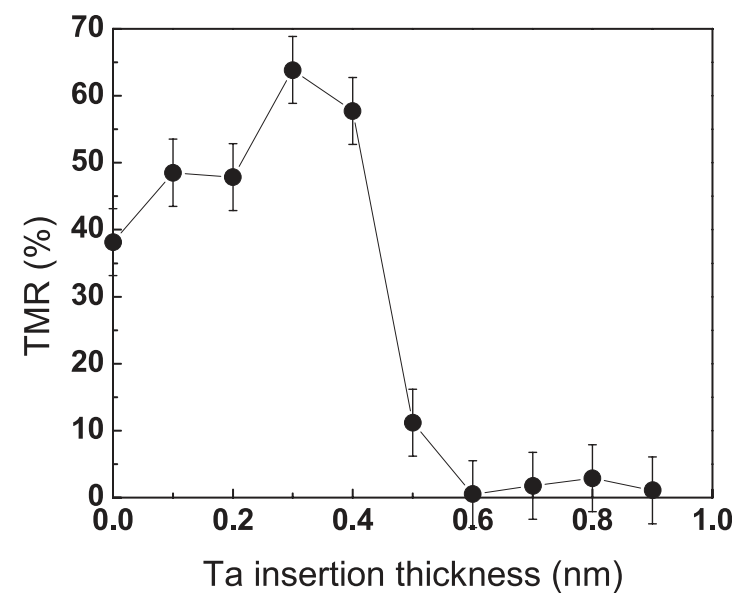

FIG. 5. TMR evolution as a function of the Ta insertion thickness.

layer. Figure 4(b) shows the evolution of the coupling field $\mathrm{H}_{\mathrm{cpl}}$ as a function of the Ta thickness. Since this coupling field is linked to the coupling energy $\mathrm{J}_{\mathrm{cpl}}$ through $\mathrm{J}=\mathrm{H}_{\mathrm{cpl}} \mathrm{M}_{\mathrm{s}} \mathrm{t}$, where $\mathrm{M}_{\mathrm{s}}$ and $\mathrm{t}$ refer to the magnetization and thickness of the top soft $\mathrm{FeCoB}$ magnetic electrode (both parameters being constant in the present case), the variation of the coupling field directly reflects that of the coupling energy. Increasing the $\mathrm{Ta}$ insertion thickness leads to a progressive decrease of the antiferromagnetic coupling energy, as a consequence of the decreasing roughness of the $\mathrm{MgO}$ barrier thanks to the beneficial effect of the underlying Ta layer. These results agree with those recently reported. ${ }^{19}$ Above a Ta thickness of $0.5 \mathrm{~nm}$, when the magnetization of the CoFeB layer is in-plane, the coupling field remains constant at about -20 Oe. This non-zero asymptotic limit probably results from remaining stray fields from both electrodes, their magnetization not being perfectly orthogonal to each other as a consequence of interfacial roughness.

Finally, transport measurements were performed on the samples with a CIPT measurement tool. After magnetic saturation of the samples in a magnetic field of $1.5 \mathrm{kOe}$, the electrical resistance of the samples was measured with applied magnetic fields of $+/-150$ Oe, large enough to saturate the magnetization of the top $\mathrm{FeCoB}$ soft layer in the positive or negative perpendicular direction. The TMR ratio as a function of the Ta insertion thickness is presented in Figure 5. The TMR variation confirms the magnetic results that have been shown previously. The TMR ratio is around $40 \%$ without $\mathrm{Ta}$ insertion, and increases with increasing Ta thickness up to $70 \%$ for a $\mathrm{Ta}$ thickness of $0.4 \mathrm{~nm}$. This reflects the improvement of the texture of the $\mathrm{CoFeB}$ layer thanks to the Ta insertion. Above $\mathrm{x}_{\mathrm{Ta}}=0.45 \mathrm{~nm}$, the TMR ratio abruptly decreases, which agrees with the reorientation of the $\mathrm{CoFeB}$ magnetization direction from perpendicular to in-plane deduced from the magnetic measurements. As a matter of fact, switching from up to down the magnetization of the soft top magnetic layer does not modify the angle it makes with that of the now in-plane bottom electrode $\left(90^{\circ}\right)$, leading to zero magnetoresistance.

In conclusion, we show in this letter a clear correlation between magnetic and transport properties of perpendicular magnetic tunnel junctions when a thin Ta layer is inserted in the hardest magnetic electrode in order to structurally decouple the growth of the $\mathrm{CoFeB}$ layer from that of the 
underlying $\mathrm{Co} / \mathrm{Pt}$ multilayer. The Ta insertion reveals its great efficiency up to $0.45 \mathrm{~nm}$, leading to an increase of the TMR ratio from $40 \%$ to $70 \%$. Above that critical Ta thickness, TMR abruptly decreases due to the reorientation of the magnetization of the CoFeB layer from perpendicular-toplane to in-plane, as a consequence of its magnetic decoupling from the $\mathrm{Co} / \mathrm{Pt}$ multilayer. A good compromise can be found for a Ta thickness around $0.4 \mathrm{~nm}$. For such a thickness, it is possible to decouple the growth of $\mathrm{CoFeB}$ from the underlying multilayer while maintaining perpendicular anisotropy of the whole structure, both conditions leading to high TMR values.

We acknowledge the financial support from the ANR (French National Research Agency) under project ANRNANO PATHOS and from the European Union under the ERC HYMAGINE project n ${ }^{\circ} 246942$.

${ }^{1}$ W. H. Butler, X.-G. Zhang, T. C. Schulthess, and J. M. MacLaren, Phys. Rev. B 63, 054416 (2001).

${ }^{2}$ S. Yuasa, T. Nagahama, A. Fukushima, Y. Suzuki, and K. Ando, Nature Mater. 3, 868-871 (2004).

${ }^{3}$ S. S. P. Parkin, C. Kaiser, A. Panchula, P. M. Rice, B. Hughes, M. Samant, and S.-H. Yang, Nature Mater. 3, 862-867 (2004).

${ }^{4}$ D. D. Djayaprawira, K. Tsunekawa, M. Nagai, H. Maehara, S. Yamagata, N. Watanabe, S. Yuasa, Y. Suzuki, and K. Ando, Appl. Phys. Lett. 86, 092502 (2005).
${ }^{5}$ O. G. Heinonen and D. V. Dimitrov, J. Appl. Phys. 108, 014305 (2010).

${ }^{6}$ D. C. Worledge, G. Hu, D. W. Abraham, J. Z. Sun, P. L. Trouilloud, J. Nowak, S. Brown, M. C. Gaidis, E. J. O'Sullivan, and R. P. Robertazzi, Appl. Phys. Lett. 98, 022501 (2011).

${ }^{7}$ K. Mizunuma, M. Yamanouchi, S. Ikeda, H. Sato, H. Yamamoto, H.-D. Gan, K. Miura, J. Hayakawa, F. Matsukura, and H. Ohno, Appl. Phys. Express 4, 023002 (2011).

${ }^{8}$ S. Monso, B. Rodmacq, S. Auffret, G. Casali, F. Fettar, B. Gilles, B. Dieny, and P. Boyer, Appl. Phys. Lett. 80, 4157-4159 (2002).

${ }^{9}$ B. Rodmacq, A. Manchon, C. Ducruet, S. Auffret, and B. Dieny, Phys. Rev. B 79, 024423 (2009).

${ }^{10}$ H. Yamamoto, J. Hayakawa, K. Miura, K. Ito, H. Matsuoka, S. Ikeda, and H. Ohno, Appl. Phys. Express 5, 053002 (2012).

${ }^{11}$ A. Natarajarathinam, R. Zhu, P. B. Visscher, and S. Gupta, J. Appl. Phys. 111, $07 \mathrm{C} 918$ (2012).

${ }^{12}$ X. Kozina, S. Ouardi, B. Balke, G. Stryganyuk, G. H. Fecher, C. Felser, S. Ikeda, H. Ohno, and E. Ikenaga, Appl. Phys. Lett. 96, 072105 (2010).

${ }^{13}$ V. Sokalski, M. T. Moneck, E. Yang, and J.-G. Zhu, Appl. Phys. Lett. 101, 072411 (2012).

${ }^{14}$ D. C. Worledge and P. L. Trouilloud, Appl. Phys. Lett. 83, 84-86 (2003).

${ }^{15}$ J. Moritz, F. Garcia, J.-C. Toussaint, B. Dieny, and J.-P. Nozières, Europhys. Lett. 65, 123-129 (2004).

${ }^{16}$ L. E. Nistor, B. Rodmacq, S. Auffret, A. Schuhl, M. Chshiev, and B. Dieny, Phys. Rev. B 81, 220407 (2010).

${ }^{17}$ L. E. Nistor, B. Rodmacq, C. Ducruet, C. Portemont, I. L. Prejbeanu, and B. Dieny, IEEE Trans. Magn. 46, 1412 (2010).

${ }^{18}$ H. X. Yang, M. Chshiev, B. Dieny, J. H. Lee, A. Manchon, and K. H. Shin, Phys. Rev. B 84, 054401 (2011).

${ }^{19}$ T. Liu, J. W. Cai, and L. Sun, AIP Advances 2, 032151 (2012). 